\title{
Smart Train Collision Detection System using a Microcontroller
}

\author{
Dogan Ibrahim \\ Department of Computer \\ Information Systems \\ Faculty of Economic \& \\ Administrative Sciences \\ Near East University, \\ Nicosia, Cyprus
}

\begin{abstract}
Train accidents occur as a result of human errors or mechanical faults in trains, in tracks, or in the signalling system. Major and costly train accidents occur due to head-on collision of trains running on the same track towards each other. Several schemes have been proposed by researchers in the past to detect the risk of possible collision and to take preventive measures. The aim of this paper is to design a novel microcontroller based system using RFID, GPS, and an RF transmitter/receiver module to detect possible collisions and inform the drivers when trains travel on the same track.
\end{abstract}

\section{General Terms}

Train collision

\section{Keywords}

Train collision detection, Microcontroller based design, RFID, GPS, Radio modem

\section{INTRODUCTION}

Train accidents are usually highly costly in terms of lost or injured human lives, damaged mechanical parts, and cancellation of normal train services. Because of this, most railway companies invest large sums of money to detect and avoid such accidents at all costs.

In general, train to train collisions can be classified into three: head-on (or head-to-head), rear-to-end, and side-to-side collisions. Head-on collision is the most dangerous one and it occurs when two trains travel on the same track towards each other. Head-on collisions usually result in heavy losses in human lives, damage to large parts of the trains and damaged hardware. Rear-to-end collision occurs when two trains travel on the same track, one following the other one, and where the speed of the rear one is greater than the speed of the one in the front. Rear-to-end accidents are less costly both in term of human lives and damaged hardware. Side-to-side collisions are in general less rare and they occur if part of a railway is damaged and as a result two trains on adjacent railways become very close to each other and collide, causing damage to the equipment and possible injury to the people on both trains. In addition, there are other common forms of collisions and accidents, for example collisions due to derailment and more commonly collisions at level crossing gates, and accidents when the railways are obstructed by large foreign objects such as trees.

In 2014, there were 1928 persons killed or seriously injured in railway accidents in the European Union (EU). A total of 2213 significant train accidents were registered in the same year in EU. Table 1 shows [1] a breakdown of the number of persons killed and injured in railway accidents in the year 2014 in EU. Train accident rates are much higher in some other countries. For example, 25,006 people died and 3,882 people were injured in a total of 28,360 railway accidents alone in India in the year 2014 [2]. When the casualties are so high it is very important that reliable and safe collision detection and prevention systems should be developed to prevent such casualties.

Perhaps the best way to avoid train collision accidents is by implementing an automatic system that can warn the drivers if there is the danger of a collision. Such systems are known as collision detection systems as they do not necessarily prevent collisions to occur. Another type of safety system is automatic collision detection and prevention system where in addition to detecting a possible collision, the system automatically shuts down (e.g. the trains stop) to prevent the collision.

In this paper, the design of a novel microcontroller based system is described which helps to detect possible train collisions. The designed system, called Train Collision Detection System (TCDS) uses RFID to detect the unique track ID that a train is travelling on, a GPS to know the exact positions and speeds of other nearby trains, a powerful RF transmitter/receiver module for communication between the trains, a buzzer to warn the drivers of any risk of collision, and a medium performance microcontroller that is used to coordinate all activities in the system.

\section{RELATED WORK}

Many different ideas have been proposed in the past by many researchers and also several practical solutions have been implemented in order to avoid the risk of collision. The problem is that all of these systems have disadvantages and do not provide a complete safe solution.

The ACD (Anti Collision Device) developed and used by Konkan Railway Corporation [3] is based on GPS positioning and track identification. The problem with the ACD system is that it is based on standard GPS positioning where the accuracy of the GPS system is not good enough for the detection of railway tracks separated by a distance of 10-15 feet. Differential GPS could have been used to improve the positioning accuracy and hence the track identification process but such additional parts increase the cost of the overall system with little improvement on the practical application. Indian Railways have successfully piloted ACDs in their northeast frontier railway, covering $1,736 \mathrm{~km}$.

Some other researchers proposed train accident prevention systems based on using wireless sensors such as RFID for detecting the track that a train is travelling on, and then using 
Table 1. Number of persons killed and injured in railway accidents in EU (2014)

\begin{tabular}{|c|c|c|c|c|c|c|c|c|c|c|c|c|}
\hline \multirow[b]{3}{*}{ Type of accident } & \multicolumn{12}{|c|}{ Number of persons } \\
\hline & \multicolumn{4}{|c|}{ Killed } & \multicolumn{4}{|c|}{ Seriously Injured } & \multicolumn{4}{|c|}{ Total } \\
\hline & 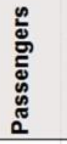 & 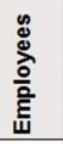 & 忘 & $\begin{array}{l}\text { एँّ } \\
\text { 。 }\end{array}$ & 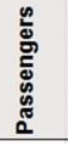 & 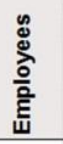 & ङે & 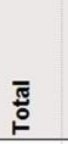 & 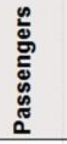 & \& & ङ્ & 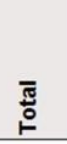 \\
\hline Collisions & 0 & 0 & 3 & 3 & 12 & 11 & 9 & 32 & 12 & 11 & 12 & 35 \\
\hline Derailments & 0 & 1 & 0 & 1 & 4 & 4 & 0 & 8 & 4 & 5 & 0 & 9 \\
\hline Accidents involving level-crossings & 1 & 2 & 291 & 294 & 21 & 9 & 314 & 344 & 22 & 11 & 605 & 638 \\
\hline Accidents to persons caused by rolling stock in motion & 14 & 22 & 665 & 701 & 78 & 22 & 395 & 495 & 92 & 44 & 1060 & 1196 \\
\hline Fires in rolling stock & 0 & 0 & 2 & 2 & 0 & 4 & 0 & 4 & 0 & 4 & 2 & 6 \\
\hline Others & 0 & 2 & 3 & 5 & 9 & 15 & 15 & 39 & 9 & 17 & 18 & 44 \\
\hline Total & 0 & 0 & 0 & 1006 & 0 & 0 & 0 & 922 & 0 & 0 & 0 & 1928 \\
\hline
\end{tabular}

A Zig-Bee and microcontroller based anti-collision system is described by Sambamurthy \& Ahammad [4] where the system gets data from moving trains, from the control center, and from the signalling posts. The proposed system is said to prevent head-on and rear-end collisions.

A surveillance based accident prevention system is described by Geethanjali et al [5] where each locomotive is equipped with an automated surveillance system. The train tracks are segmented and each segment is given unique identifiers. The surveillance system inside the locomotive reads the track identifiers and this information is shared with neighbour trains using RF communication. The system compares its own track identifier with neighbour track identifiers and if same track identifiers are found then the concerned motorman is cautioned to stop the train.

Oh et al [6] describe an automatic train protection simulator for radio-based train control systems which helps simulate safety analysis and evaluation of automatic train protection. An algorithm is presented by the authors for automatic train protection.

Chellaswamy et al [7] describe an intelligent collision avoidance system (ICAS) for locomotives for avoiding headon collisions. The proposed system avoids collision by notifying the status of two trains on the same track and by giving a sound alert. A vibration sensor is used to identify a train on a track. The prototype system of the authors was designed using a microcontroller and was tested using wireless communication.

Zhang et al [8] describe performance analysis of fast RFID tags used to monitor and regulate the railway system. Collisions due to insufficient reading times of the RFID readers are discussed and alternative solutions are given in the paper.

Garcia et al [9] give an overview and comparison of the state of the art in collision avoidance systems related to transportation and its applicability to rail transportation. The proposed system relies on position detection and direct communication. The paper focuses on the communication aspects where different communications layers are described in detail.

\section{THE DEVELOPED SYSTEM}

\subsection{The Hardware}

The block diagram of the system developed by the author is shown in fig. 1. This is a smart intelligent microcontroller based system, having the following major hardware components:

\subsubsection{Clicker 2 for PIC18FJ}

This is a microcontroller development system [10] using the PIC18F87J50 type medium performance 8-bit microcontroller. The microcontroller is clocked from an $8 \mathrm{MHz}$ external crystal which is multiplied using an internal PLL to the operating frequency of $32 \mathrm{MHz}$. The development board contains two mikroBUS sockets where various Click boards can be connected to. Power to the board is supplied through a mini USB cable. The microcontroller is programmed through a serial port facilitated by Bootloader program resident in the program memory. Access to the I/O ports is via the connectors at both sides of the board. The basic features of the PIC18F87J50 microcontroller are:

- 128 Kbyte flash program memory

- 3904 byte SRAM memory

- $65 \mathrm{I} / \mathrm{O}$ pins

- 12 channel 10-bit ADC converters

- 3 PWM channels

- $\quad$ SPI and I2C channels

- 2 serial UARTs

- 2 8-bit, 3 16-bit timers

- Internal oscillator

- $3.3 \mathrm{~V}$ low power operation

\subsubsection{GPS Click}

This is a mikroBUS compatible GPS module [11] using the LEA-6S high performance GPS chip, having $-136 \mathrm{dBm}$ tracking sensitivity, and capable of tracking up to 16 satellites. The module operates with $+3.3 \mathrm{~V}$ and interface to the module is via USART serial port operating at 9600 baud. The NMEA sentence \$GPRMC was used in the design since this sentence gives the speed in addition to the positional data.

The \$GPRMC sentence gives the following information: 
- $\quad$ Latitude and longitude with directions and status

- $\quad$ Speed

- $\quad$ Track angle

- $\quad$ Date and time

- Magnetic variation
The GPS click module is connected to mikroBUS socket 2 of the microcontroller development board.

The basic features of the GPS Click board are:

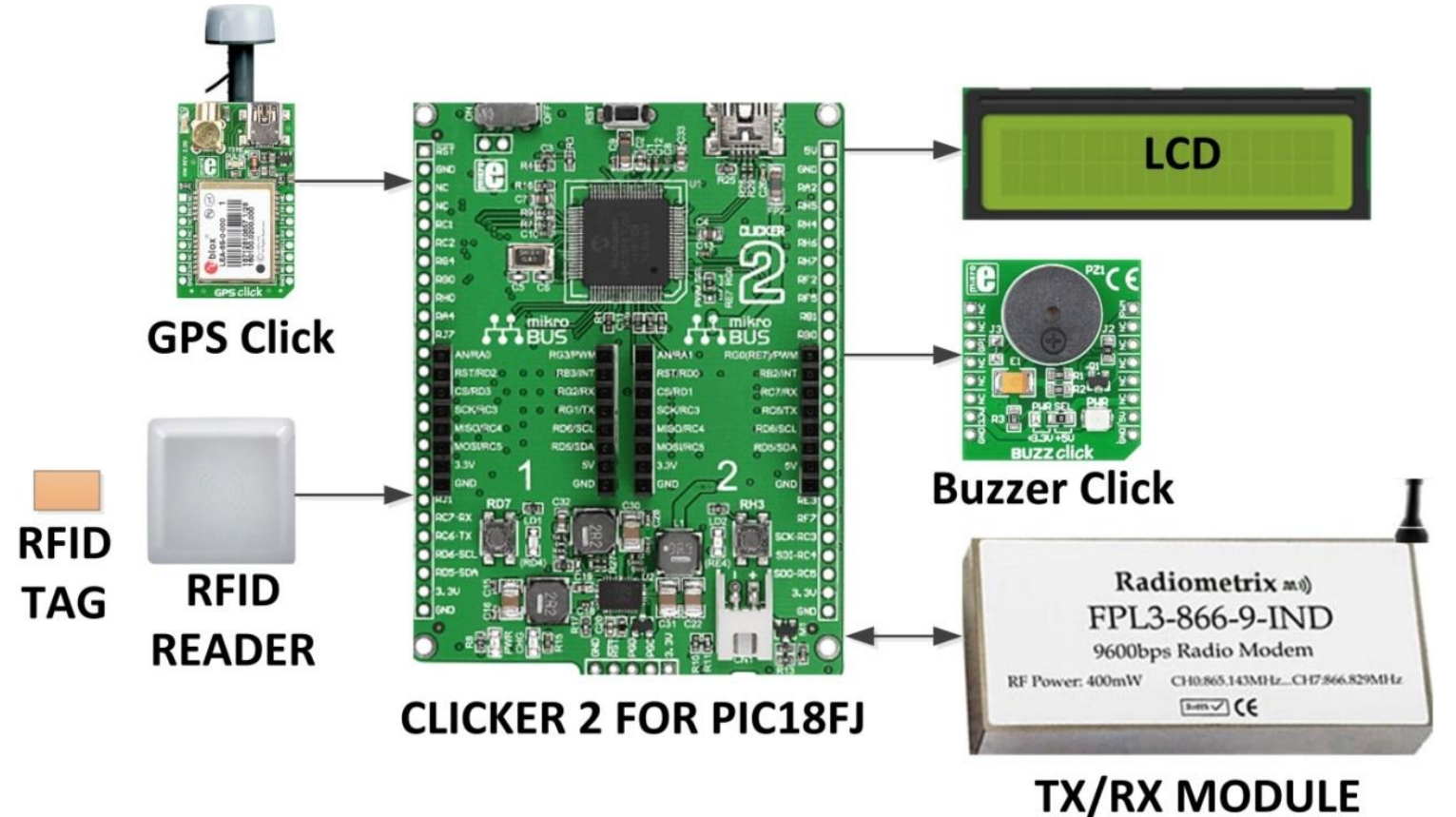

Fig. 1: Block diagram of the developed system

- $\quad$ Tracking up to 16 satellites

- $\quad$ UART and I2C interface

- $\quad 3.3 \mathrm{~V}$ low power operation

- $\quad-160 \mathrm{dBm}$ tracking sensitivity

- Support for active antenna

Operation from an active external antenna mounted on top of the train is an important requirement of the GPS in this design since the system is to be mounted inside a train and strong satellite signals may be required since satellite view could be obstructed.

\subsubsection{LCD}

A serial LCD is used in the design for simplicity. The LCD displays information related to the state and safety of the system.

\subsubsection{Radio Modem Module}

Long range operation is a fundamental requirement in this design and because of this the FPL3-866-9 type radio modem module from Radiometrix [12] is used. This is a professional quality UHF radio modem with an RF output power of $400 \mathrm{~mW}$, having a line of sight range of over $3 \mathrm{~km}$ (this range should be enough if $1 \mathrm{~km}$ minimum safe distance to avoid collision is assumed when the trains are travelling at the maximum speed of $80 \mathrm{~km} / \mathrm{hr}$ ). The module is interfaced to the microcontroller through a USART serial port operating at 9600 baud.

The basic features of the FPL3-866-9 TX/RX module are:
- UHF operation

- Serial modem at 9600 baud

- $\quad+26 \mathrm{dBm}, 400 \mathrm{~mW}$ RF power

- $\quad-107 \mathrm{dBm}$ receiver sensitivity

- Usable range over $3 \mathrm{~km}$

- 8 channels

- $\quad 400 \mathrm{~mA}$ current consumption (at full power)

- Operation from $+5 \mathrm{~V}$

\subsubsection{RFID Reader}

The RFID reader used in the design is the professional quality PK-UHF101-1 passive reader [13]. Because the RFID reader is to be used outside, the main requirements are:

- The reader should be able to pick up signals when the passive tags are at several metres away. This is necessary since the reader is underneath the locomotive while the RFID tags are mounted on the sleepers between the rails

- The operating temperature range of the reader should be wide

- The reader should be sturdy and have wide operating temperatures

- The reader should be reliable

PK-UHF101-1 reader operates at $865-868 \mathrm{MHz}$ UHF frequency and provides an output power of $30 \mathrm{dBm}$. With the 
built-in $8 \mathrm{dBi}$ polarised antenna the tag reading range is around 5 metres. Although the reader offers RS232, RS485 and Wiegand 26/34 protocols, the RS232 interface was used in the design.

The basic features of the PK-UHF101-1 RFID reader are:

- $\quad 0-30 \mathrm{dBm}$ power output

- 5 - 8 metre reading range

- Adjustable frequency range

- $\quad$ Built-in $8 \mathrm{dBi}$ polarised antenna

- $\quad$ Reader and writer functions

- $\quad-20^{\circ} \mathrm{C}$ to $+70^{\circ} \mathrm{C}$ operating temperature

- $\quad+9 \mathrm{~V}$ operation

- Sturdy design

\subsubsection{RFID Tags}

These are passive tags that are available in many different shapes and sizes. The tags can be programmed to return the required codes using the PK-UHF101-1 RFID reader module. Since the tags will be mounted on the sleepers between the rails, it is important to use tags which are sturdy and can withstand to high and low temperatures and are also waterproof.

\subsubsection{Buzzer Click}

This is a microBUS compatible board [14] having a small transistor operated buzzer on-board. The buzzer is used as a warning device and is activated if there is a risk of collision. Although a small buzzer is used in the prototype design, it may be a better idea to use a speaker with higher decibel sound output in a real design.

\subsubsection{Antennas}

Both the GPS module and the radio modem module require external antennas. An active antenna was connected to the GPS module so that even weak signals could be picked up. Similarly, an end fed dipole antenna was connected to the radio modem module for long range of operation. In general, the range of transmission can be increased using directional antennas but that may not be required in this application.

Additional components such as relays could also be used in the design to activate the electrical braking system at a suitable time in case there is the risk of a collision. This depends upon the type of breaking system used on the trains.

\subsection{The Operation}

The operation of the developed train collision detection system is shown diagrammatically in fig. 2. The RFID tags are placed on the railway sleepers, each having a unique identification number that identifies the track it is placed on. A train knows the track it is travelling on by reading the track identity numbers from the RFID tags. The latitude, longitude, and speed of a train are determined by the on-board GPS module. A train transmits its RFID tag number, latitude, longitude and speed through the radio modem module at frequent intervals (e.g. every minute). At the same time, the radio modem module receives similar information from other trains within the $3 \mathrm{~km}$ range. A train compares its own RFID tag number with the received tag number and if they are different then the two trains are assumed to be on different tracks of the railway. If this is the case then there is no risk of collision and no further action is taken. If on the other hand the two tag numbers are the same then the trains are assumed to be on the same track and further processing is required to find out whether or not there is the risk of collision. At this point, there are several possibilities:

- The two trains are travelling towards each other in which case there is very strong possibility of a headon collision. This situation is very important and it must be determined reliably with no errors.

- The two trains are following each other in which case there could be collision depending upon the relative speeds of the trains. If the speed of the rear train is greater than the speed of the front train then there is the possibility of rear-to-end collision and further investigation is required. If on the other hand the speed of the rear train is less than the speed of the front train then there is no possibility of collision even though the two trains are on the same track. If the latter is the case then no further action is necessary.

- The last possibility is that the two trains are on the same track but are departing from each other, in which case there is no possibility of collision and again no further action is necessary. 


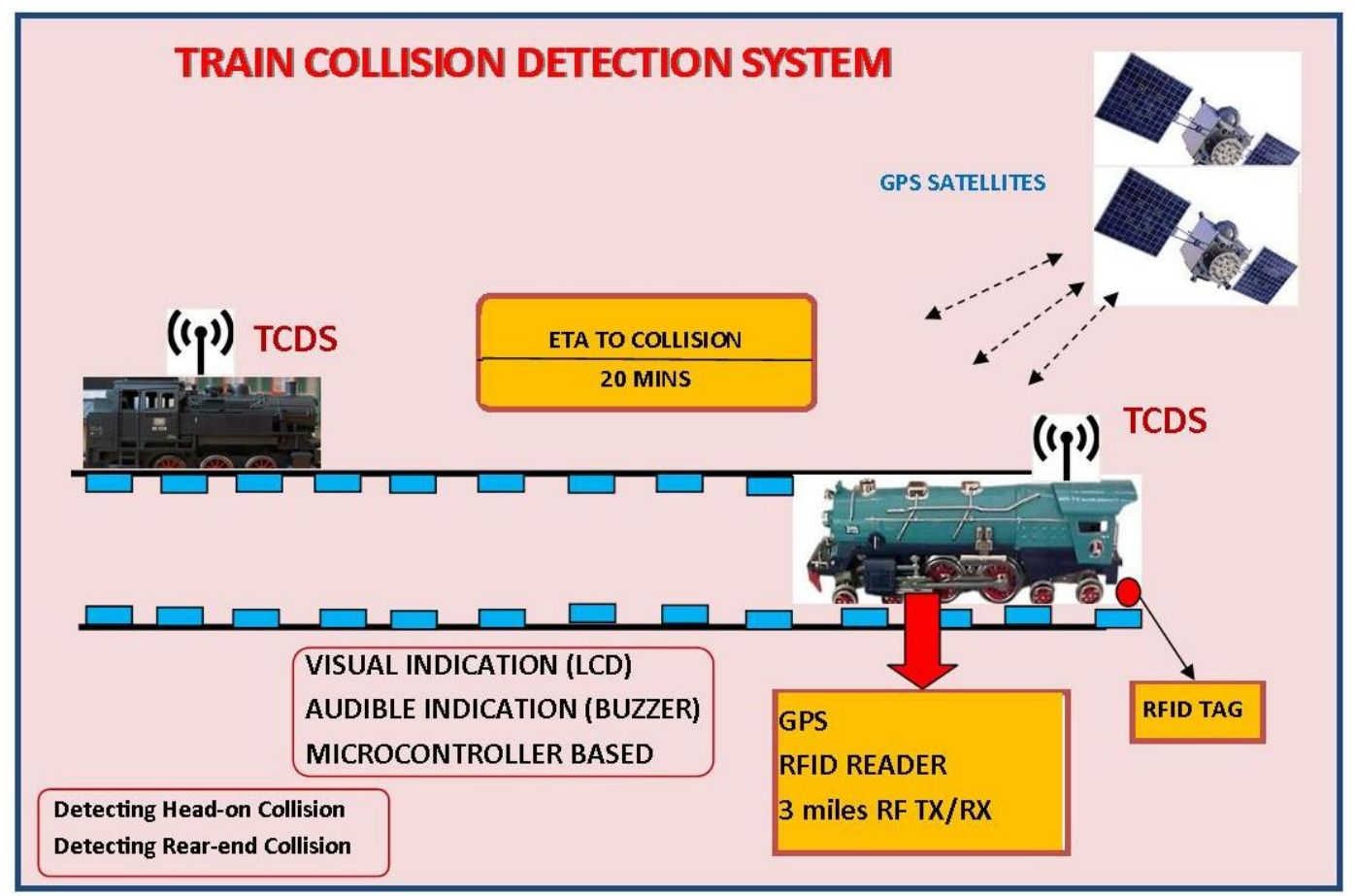

Fig. 2: Operation of the TCDS system

\subsection{The Software}

The microC Pro for PIC language and the IDE was used during the development of the software. This is a professional $\mathrm{C}$ compiler for the PIC microcontrollers, having large number of libraries that support interfaces such as RS232, I2C, SPI, USB, Ethernet, CAN bus, LCD, GLCD, buttons, and many more. In addition, a software simulator and a hardware debugger (mikroICD) are provided to help simulate and test the program during its development cycle. The developed program is loaded to the target microcontroller program memory using a Bootloader program on the PC.

The RFID reader routine, radio modem module receive routine, and the GPS receive routine have all been implemented in interrupt service routines since the data coming from these sources are asynchronous and have to be serviced as soon as new data becomes available. PIC18 series of microcontrollers vector the interrupts to the same address and therefore it is necessary to determine the interrupting source before any action can be taken.

The software implements a simple algorithm to find out whether or not there is the risk of a collision and if so, the time to the collision is calculated using the relative speeds and distance between the trains and this time is displayed on the LCD. At the same time the buzzer sounds to warn the driver of the coming danger. Both the head-on and rear-to-end types of collisions are analysed by the software.

\subsubsection{The Collision Algorithm}

The collision detection algorithm is described below for the case when two trains happen to be on the same track:

Assume that two trains TR1 and TR2 have speeds S1 and S2 $\mathrm{km} / \mathrm{hr}$ and are at a distance of $\mathrm{d} 1 \mathrm{~km}$ from each other as shown in fig. 3 . The distance d1 between the two trains is calculated from knowledge of their GPS positions.

Assume that another measurement is made after $\mathrm{T}$ minutes (e.g. one minute). Let $\mathrm{d} 2$ be the new calculated distance between the two trains, and also assume that the speed of the trains do not change during time $\mathrm{T}$. The following process is repeated at regular intervals:

Case 1: If $\mathrm{d} 2>\mathrm{d} 1$ then there is no risk of collision.

Case 2: if $\mathrm{d} 2<\mathrm{d} 1$

Case 2.1: Head-on collision will occur if:

$$
\mathrm{d} 2=\mathrm{d} 1-(\mathrm{S} 1+\mathrm{S} 2) \mathrm{T} / 60
$$

and the estimated time to collision (ETC) is given by:

$$
\mathrm{ETC}=\mathrm{d} 2 /(\mathrm{S} 1+\mathrm{S} 2)
$$

Case 2.2: if otherwise $\mathrm{S} 1<\mathrm{S} 2$ and

$$
\mathrm{d} 2=\mathrm{d} 1+(\mathrm{S} 2-\mathrm{S} 1) \mathrm{T} / 60
$$

then the two trains are following each other with no risk of collision

Case 2.3: if otherwise $\mathrm{S} 1>\mathrm{S} 2$ and

$$
\mathrm{d} 2=\mathrm{d} 1-(\mathrm{S} 1-\mathrm{S} 2) \mathrm{T} / 60
$$

then the two trains are following each other and there is risk of Rear-to-end collision with the estimated time to collision (ETC) given by:

$$
\mathrm{ETC}=\mathrm{d} 2 /(\mathrm{S} 1-\mathrm{S} 2)
$$

\subsection{The Prototype System}

The prototype system was designed and constructed on a large breadboard and is shown in fig. 4. Two such prototype systems were constructed so that operation of the algorithm could easily be tested and verified. The overall system was tested in a laboratory environment with the two systems placed away from each other.

\section{FUTURE SCOPE}

The developed system can be enhanced further by adding automatic emergency help line where the position of a train can be sent to a help center with pre-recorded messages after an accident, using perhaps the GPRS or a similar technology. 
Additionally, movements of the trains and their speeds can be stored on an SD card in real-time for future analysis, just like the Blackbox of an aircraft. Finally, the system can be linked to the train brake system to halt the train should there be risk of collision in a pre-defined future time or distance.

\section{REFERENCES}
[1] Railway
Safety
Statistics,

http://ec.europa.eu/eurostat/statistics-

explained/index.php/Railway_safety_statistics

[2] Indian National Crime Records Bureau, www.ncrb.gov.in

[3] About Indian Railways, http://indianrailways.gov.in/railwayboard/view_section.js $\mathrm{p}$ ?lang $=0$ \&id $=0,1$

[4] Sambamurthy, N., Ahammad, H. "Prevention of train accidents Using Wireless Sensor Networks", Int. Journal of Engineering Research and Applications, ISSN : 22489622, Vol. 3, Issue 6, Nov-Dec 2013, pp.1592-1597

[5] Geethanjali, M., Shamanthan, L.D.V.S.,Krishnan, K.P.S., Raji, G. "RF Based Train Collision Avoidance System", Annual IEEE India Conference (INDICON) , 13-15 December 2013.

[6] Oh, S., Yoon, Y., Kim, Y. "Automatic Train Protection Simulation for Radio-based Train Control System",
International Conference on Information Science and Applications (ICISA), 23-25 May, 2012.

[7] Chellaswamy, C., Arul, S., Balaji, L. "Design and Analysis of an Intelligent Collision Avoidance System for Locomotives", International Conference on Sustainable Energy and Intelligent Systems (SEISCON), 20-22 July, 2011

[8] Zhang, X., Lakafosis, V., Traille, A., Tentzeris, M. "Performance Analysis of Fast Moving RFID Tags in State-of-the-art High-speed Railway Systems", IEEE International Conference on RFID-Technology and Applications (RFID-TA), 17-19 June, 2010.

[9] Garcia, C.R., Lehner, A., Strang, T., Rockl, M. "Comparison of Collision Avoidance Systems and Applicability to Rail Transport", $7^{\text {th }}$ International Conference on ITS (ITST'07), 6-8 June, 2007.

[10] Clicker 2 for PIC18FJ user Guide, www.mikroe.com

[11] GPS Click User Guide, www.mikroe.com

[12] FPL3-866-9 User Guide, www.radiometrix.com

[13] PK-UHF101-1 User Guide, www.pongee.com

[14] Buzzer Click User Guide, www.mikroe.com

\section{APPENDIX}

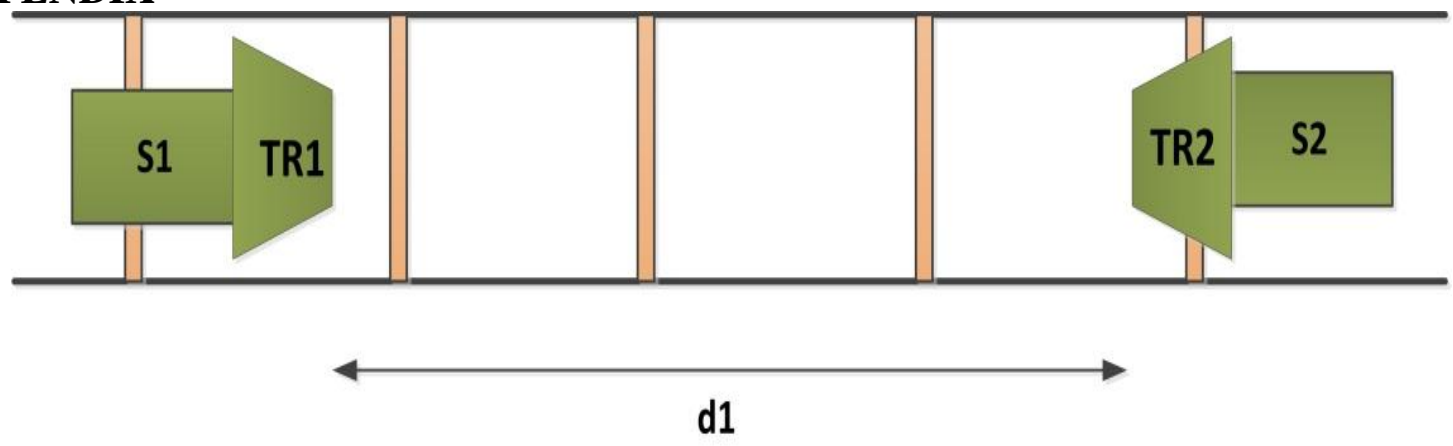

\section{After time T:}

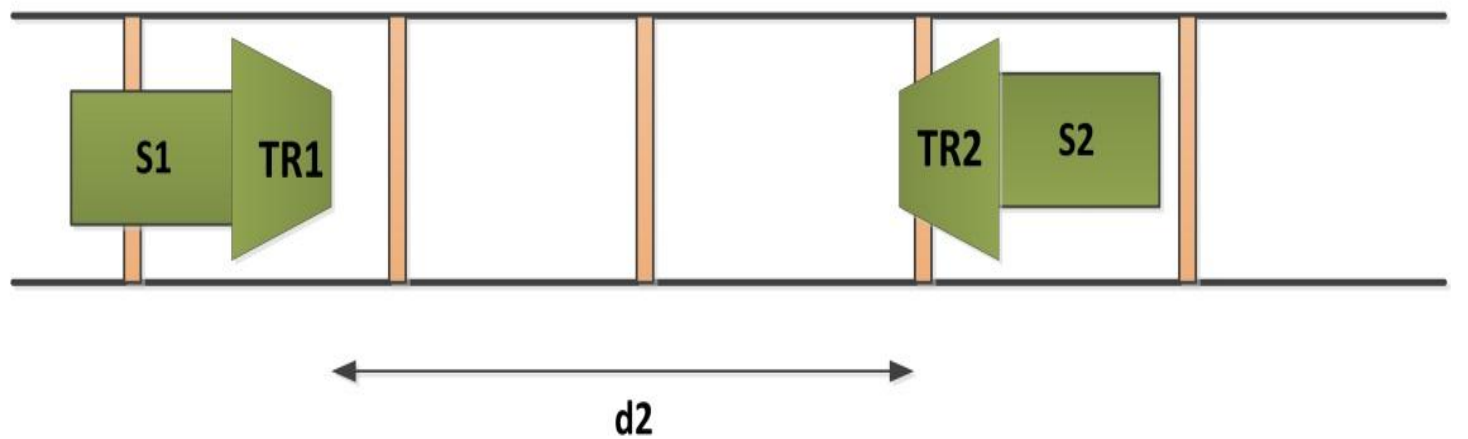

Fig. 3: Two trains on the same track 


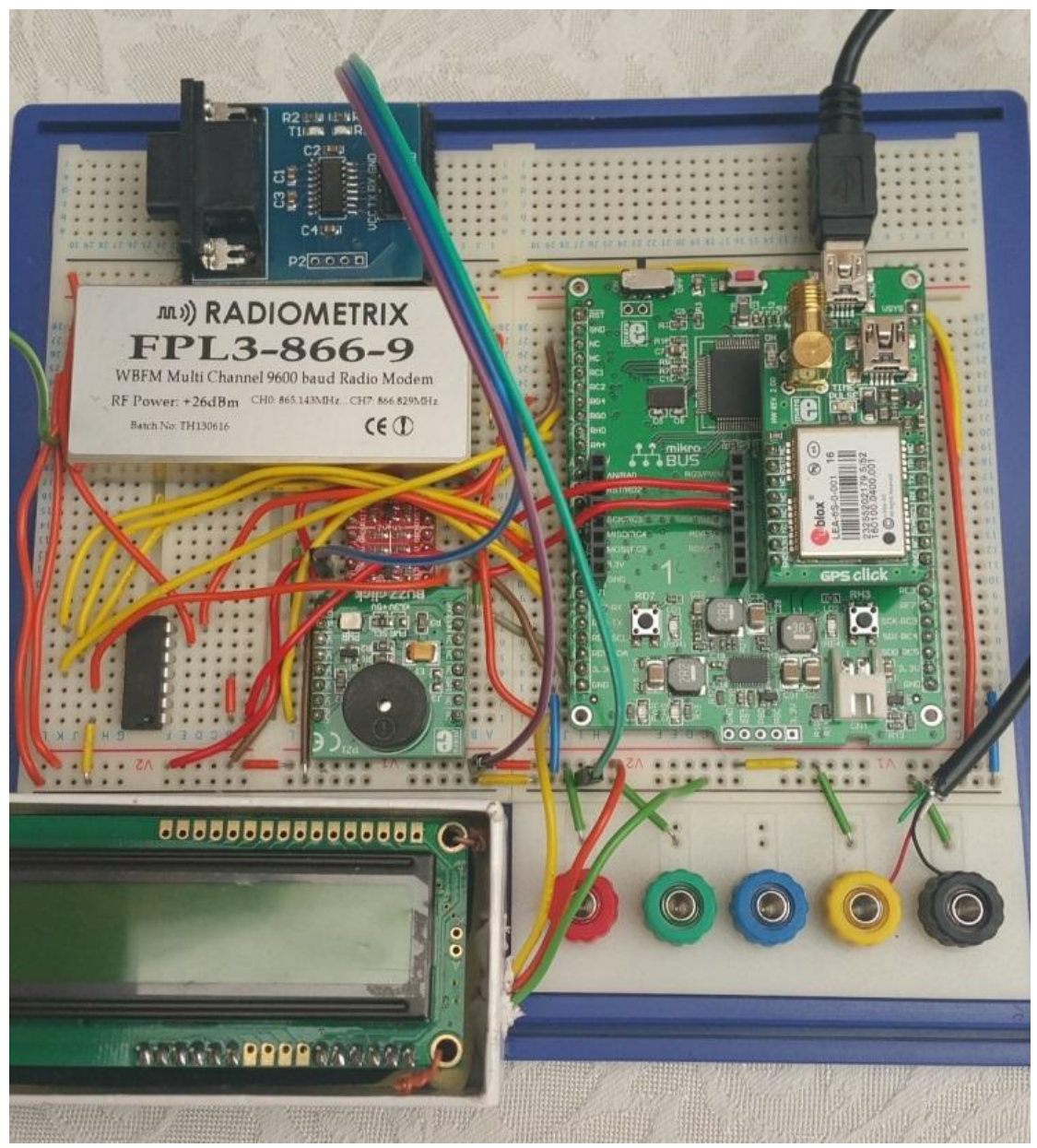

Fig. 4: Developed system on a breadboard 